\title{
Evolución clínica y de laboratorio de episodios de neutropenia febril en niños con cáncer, en un hospital de Colombia, período 2007-2009
}

\author{
Isabel C. Hurtado, Diana P. Sánchez, David A. Espinal y Carlos Garcés
}

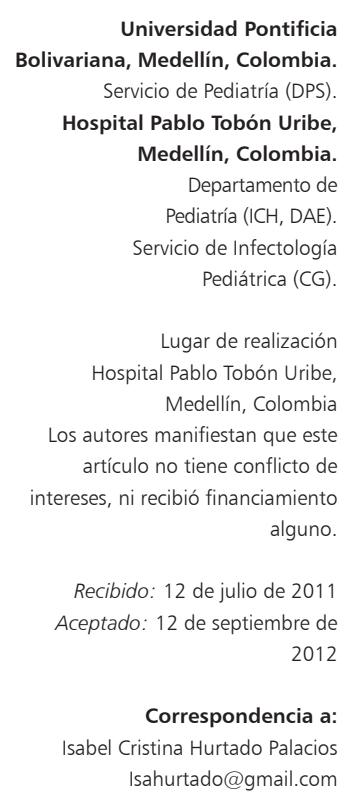

\section{Introducción}

$\mathrm{L}$ a terapia antineoplásica en los pacientes pediátricos tiene un alto impacto en la inmunidad, tanto innata como adquirida y en la respuesta fisiológica a la infección, disminuyendo la respuesta específica y no especifica frente a los patógenos, lo que conlleva una alta susceptibilidad a infecciones, principalmente bacterianas y fúngicas ${ }^{1}$. La neutropenia definida como un recuento total de neutrófilos menor a 500 céls $/ \mathrm{mm}^{3}$ o menor a 1.000 céls $/ \mathrm{mm}^{3}$ cuando hay una alta posibilidad de disminución rápida, es una de las complicaciones más comunes en los niños tratados por patologías malignas, siendo el principal parámetro para determinar el riesgo de infección ${ }^{2}$. Por otra parte, los signos y síntomas clínicos de infección en estos pacientes pueden ser escasos; en la mayoría de las ocasiones, la fiebre es la principal o única manifestación de la misma, por lo cual todo paciente neutropénico con fiebre se debe considerar con una potencial infección grave² ${ }^{2}$.

Los estudios epidemiológicos revelan que sólo 10 a $30 \%$ de los episodios de neutropenia febril (NF) presentan comprobación microbiológica de la infección siendo más común las causas bacterianas. Según datos extractados del Consenso de la Sociedad Latinoamericana de
Infectología Pediátrica publicado en el año 2011, en 15 a $25 \%$ de los niños afectados por NF se comprobará la existencia de bacteriemia, y en otro porcentaje similar, de infecciones bacterianas localizadas ${ }^{2}$. La mayoría de ellas serán causadas por microorganismos multi-resistentes, ya que los pacientes suelen haber tenido hospitalizaciones recientes y tratamientos previos con antimicrobianos de amplio espectro ${ }^{4,5}$.

Los virus se han logrado identificar en 25 a $37 \%$ de los episodios de NF, siendo el tipo de virus muy variable pero en la mayoría de los casos son infecciones transitorias y de resolución espontánea ${ }^{6}$. Los demás patógenos -fúngicos y parasitarios- son menos comunes y se asocian a características especiales ${ }^{1}$.

Conocer los agentes etiológicos más frecuentes como también su susceptibilidad a los antimicrobianos en los episodios de NF es de gran importancia dado que permite escoger la terapia antimicrobiana empírica inicial de manera más racional ${ }^{1-3}$.

El pronóstico de los pacientes con NF es muy variable, por esta razón se han sugerido múltiples protocolos de tratamiento ${ }^{4,5}$, en la actualidad los más aceptados incluyen hospitalización e inicio de antibioticoterapia de amplio espectro ${ }^{4}$. Para esto se deben conocer las características 
de la población a tratar incluyendo la microbiología de los episodios en la propia institución, la duración e intensidad de la neutropenia, la localización más común de la infección, la caracterización del episodio en alto y bajo riesgo de infección grave. Las características clínicas y de laboratorio ayudan a determinar el tipo de microorganismo implicado y el antimicrobiano a elegir ${ }^{1,4}$.

Debido a la heterogeneidad de datos y al desconocimiento de las características locales nuestro objetivo fue revisar el comportamiento clínico y de laboratorio de nuestros pacientes con NF, variables importantes en el momento de definir la estratificación y la terapia empírica inicial necesaria en ellos.

\section{Pacientes y Métodos}

Se incluyeron todos los pacientes pediátricos (hasta 15 años) con diagnóstico de neutropenia febril secundaria a tratamiento antineoplásico del Hospital Pablo Tobón Uribe (HPTU) de Medellín, Colombia.

Período de estudio: enero de 2007 a diciembre de 2009.

\section{Definiciones}

Neutropenia: Recuento total de neutrofilos $<500$ céls $/ \mathrm{mm}^{3}$. Se extendió este límite a $<1.000$ céls $/ \mathrm{mm}^{3}$ en pacientes de alto riesgo, con signos de shock o si el recuento tendió a disminuir en las $48 \mathrm{~h}$ posteriores a la evaluación inicial. Fiebre: Temperatura axilar mayor a $38^{\circ} \mathrm{C}$ en dos o más tomas o mayor a $38,5^{\circ} \mathrm{C}$ en una sola toma o su equivalente en otros lugares. Riesgo: Alto, edad mayor a 12 años, paciente con leucemia en recaída o segundo tumor, intervalo entre el término del último ciclo de quimioterapia y el inicio de la fiebre $<7$ días, fiebre $>39^{\circ} \mathrm{C}$ axilar, leucocitos absolutos $<100$ céls $/ \mathrm{mm}^{3}$, plaquetas $<50.0000$ céls $/ \mathrm{mm}^{3}$ y PCR mayor a $90 \mathrm{mg} / \mathrm{L}$. Los pacientes que no cumplieran estas características se clasificaban como de bajo riesgo.

\section{Recolección de datos}

La edad, sexo, duración de la fiebre, terapia antimicrobiana inicial y final, días previos de terapia antineoplásica, recuento de neutrófilos inicial y final, resultados de hemocultivos y urocultivos, infección asociada a catéter, muerte, valores de PCR y uso de factor estimulante de colonias de granulocitos se tomaron de las historias clínicas de los pacientes incluidos en el estudio para lo cual se usaron formularios previamente diseñados.

\section{Diseño del estudio}

Estudio observacional, descriptivo, retrospectivo.

\section{Análisis estadístico}

Se analizaron los datos en SPSS-6 calculando para las variables cuantitativas medidas de tendencia central y dispersión (media, mediana, desviación estándar y percentiles) y para las variables cualitativas se construyeron tablas de frecuencias absolutas y relativas.

El presente estudio fue aprobado por el Comité de Ética del HPTU.

\section{Resultados}

En el período de tres años se registró y atendió un total de 105 episodios de NF secundaria a tratamiento antineoplásico en 83 pacientes del HPTU, de éstos 57 eran hombres (68,6\%), el promedio de edad fue de 8,19 años (DE: 3,13 años).

En 72 episodios los pacientes recibieron tratamiento antineoplásico entre 0 y 7 días antes de la neutropenia $(68,5 \%)$ (Tabla 1). En tres episodios no se conocían los días previos de quimioterapia ya que no la recibían en nuestra institución.

El diagnóstico más frecuente fue leucemia linfoide aguda, encontrada en $42 \%$ de los episodios, seguido de leucemia mieloide aguda en 9\%, linfoma no Hodgkin $7,6 \%$ y sarcomas en $14 \%$. La media de duración de la fiebre fue de 5,23 días (DE: 3,3 días), con un mínimo de 1 día y un máximo de 21 días.

La mayoría de las infecciones correspondieron a bacteriemias $(22,9 \%)$. Otros focos encontrados fueron neumonías $(12,3 \%)$, todas diagnosticadas por clínica + hallazgos patológicos en la radiografía de tórax, gastroenteritis aguda $(5,7 \%)$, infecciones de piel y tejidos blandos $(4,8 \%)$ e infecciones urinarias (2,9\%). En los demás episodios no se logró, con los exámenes practicados, identificar un foco infeccioso claro $(51,4 \%)$.

En todos los casos se tomaron hemocultivos al ingreso, siendo positivos en 24 episodios (22,9\%); las bacterias más comúnmente implicadas fueron Staphylococcus au-

\begin{tabular}{|c|c|}
\hline Variable & Frecuencia (\%) \\
\hline \multicolumn{2}{|l|}{ Sexo } \\
\hline Hombres & $57 \quad(68,6 \%)$ \\
\hline Edad promedio (DE) & 8,19 años (3,13 años) \\
\hline \multicolumn{2}{|c|}{$\begin{array}{l}\text { Días previos a partir último día de } \\
\text { tratamiento antineoplásico }\end{array}$} \\
\hline Entre 0 y 7 días & $72 \quad(68,5 \%)$ \\
\hline Entre 8 y 30 días & $20 \quad(19,0 \%)$ \\
\hline Más de 30 días & $11 \quad(10,4 \%)$ \\
\hline Desconocido & $3 \quad(2,8 \%)$ \\
\hline \multicolumn{2}{|l|}{ Diagnóstico } \\
\hline Leucemia & $58 \quad(55,2 \%)$ \\
\hline Linfoma & $16(15,2 \%)$ \\
\hline Sarcoma & $14 \quad(12,3 \%)$ \\
\hline
\end{tabular}




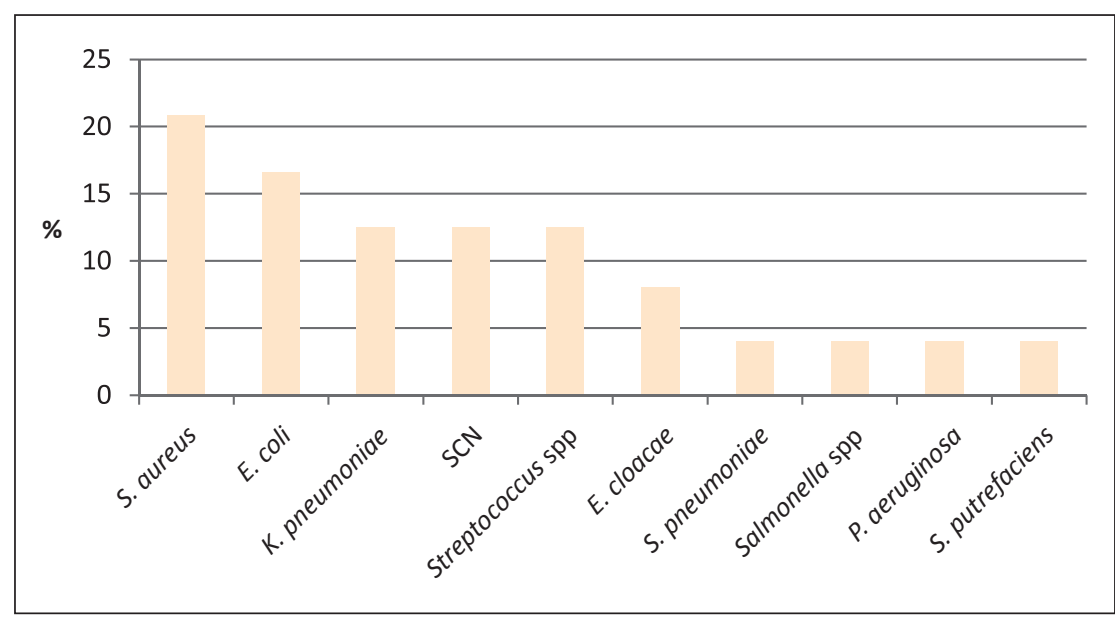

Figura 1. Microorganismo más frecuentemente aislados en hemocultivos (porcentajes) SCN: Staphylococcus coagulasa negativa.

reus sensible a meticilina que se encontró en 5 ocasiones (20,8\%) -no hubo presencia de $S$. aureus resistente a meticilina- y Escherichia coli no productora de BLEE que se aisló en 4 episodios (16,6\%). Las demás bacterias aisladas y su frecuencia de aparición se encuentran en la Figura 1.

Se hizo diagnóstico de infección asociada a catéter en 9 pacientes (8,6\%). En 39 episodios se realizó urocultivo, de éstos 5,1\% resultó positivo; las bacterias aisladas fueron E. coli y Klebsiella pneumoniae, ambas encontradas en igual proporción. En 4 ocasiones se solicitó coprocultivo encontrando como causante Salmonella spp en 3 de ellos. En un paciente se detectó antígeno para rotavirus.

Sólo en un episodio se tomó panel viral encontrándose positivo para virus respiratorio sincicial y en 3 se tomó IgM para Mycoplasma pneumoniae siendo positiva en 2 de ellos.

En todos los episodios se indicó inicialmente tratamiento antimicrobiano, el más utilizado fue piperacilina/ tazobactam que se usó en 92 ocasiones $(87,6 \%)$, seguido de meropenem que se usó en $4(3,8 \%)$; los demás antimicrobianos fueron de uso ocasional e incluyeron ampicilina/sulbactam (n: 1), metronidazol (n: 1), cefepime (n: 1) y vancomicina (n: 1$)$, otros (4\%).

La terapia antimicrobiana final fue diversa y en algunos casos incluyó más de un antimicrobiano o la asociación de antibacterianos con antimicóticos e incluso antivirales, los más comúnmente utilizados fueron piperacilina/ tazobactam en 66 ocasiones, meropenem en 19, fluconazol en 14 y vancomicina en 13 episodios. Entre las combinaciones más usadas se encontraban vancomicina + meropenem en 11 oportunidades, piperacilina/tazobactam + fluconazol en seis y piperacilina/tazobactam + aciclovir en dos episodios. Otros medicamentos fueron ganciclovir, valganciclovir, basados en carga viral positiva para CMV en dos pacientes, voriconazol en un paciente para el tratamiento de aspergilosis invasora, e isoniazida, rifampicina, etambutol y pirazinamida, como tratamiento anti-tuberculoso en un paciente que tenía criterios clínico, radiológico y tuberculínico compatibles.

Se usó factor estimulante de colonias de granulocitos como coadyuvante en el manejo de la neutropenia en 65 episodios $(61,9 \%)$.

En esta serie se observó una mortalidad de 6,7\%.

El recuento de neutrófilos inicial promedio fue de 518,4 céls $/ \mathrm{mm}^{3}$ (DE 1.149,2 céls $/ \mathrm{mm}^{3}$ ) y final de $2.679,7$ céls $/ \mathrm{mm}^{3}$ (DE 3.156,7 céls $/ \mathrm{mm}^{3}$ ), existiendo en ambos grupos valores desde 0 céls $/ \mathrm{mm}^{3}$. Los valores de proteína $\mathrm{C}$ reactiva (PCR) fueron muy variables con una media de $113 \mathrm{mg} / \mathrm{L}$ (DE $130 \mathrm{mg} / \mathrm{L})$.

\section{Discusión}

La NF es una condición frecuente en los pacientes pediátricos con cáncer, afecta a los pacientes oncológicos sin tener distinción de sexo o edad. En nuestro estudio se presentó esta complicación en una cantidad ligeramente mayor de hombres que mujeres, y en pacientes de todas las edades, lo que corrobora lo visto en otros estudios ${ }^{7,8}$. La media de edad de 8,19 años corresponde con el rango de edad usual de presentación del cáncer en pediatría ${ }^{10}$. Los principales diagnósticos de estos pacientes fueron leucemia y linfoma que son los principales tipos de cáncer en niños ${ }^{10}$; estas neoplasias requieren quimioterapia agresiva por lo que pueden infectarse y desarrollar sepsis más fácilmente 4 .

El pico de presentación de la NF en este estudio fue entre 0 y 7 días después de recibir la quimioterapia, en concordancia con lo reportado en la literatura médica (0-10 días $)^{1,9,11}$. Es importante recalcar que los pacientes incluso después de un mes o más de recibir la quimioterapia continúan en riesgo de experimentar episodios de NF aunque éste disminuye con el tiempo ${ }^{11}$.

En esta cohorte de pacientes se logró identificar el foco infeccioso en $48,6 \%$ de los episodios; al respecto, en la literatura médica reportan que hasta $50 \%$ de los pacientes tienen una infección establecida ${ }^{12}$, de éstos $22,9 \%$ de nuestra serie correspondió a bacteriemia, concordando con el rendimiento de los hemocultivos que se reporta en la literatura científica $(20-30 \%)^{1,5,12}$. El 5,1\% de los pacientes tuvo urocultivo positivo, este último no se realizó en todos los pacientes ya que según las diferentes guías sobre NF disponibles, durante la evaluación de los pacientes con NF sólo se debiera realizar exámenes de orina en pacientes con síntomas urinarios, en pacientes bajo 2 años de edad en los que no se les pudiera dilucidar la causa de la fiebre y en aquellos pacientes en los que la neoplasia estuviese ubicada en el tracto genitourinario ${ }^{1,4}$. 
En diferentes estudios se ha demostrado que los aislamientos microbiológicos en pacientes con neutropenia febril oscilan entre 10 y $30 \% 0^{1,6,12}$, lo que fue ligeramente superior en nuestro estudio (38\%). Los demás focos infecciosos correspondieron a neumonías $(12,3 \%)$, gastroenteritis aguda $(5,7 \%)$ e infecciones de piel y tejidos blandos $(4,8 \%)$; aunque en éstas no se logró identificar el microrganismo causal, se dio tratamiento apropiado gracias a la identificación del foco de infección. Estos focos están descritos en la literatura médica como focos infecciosos frecuentes en pacientes con $\mathrm{NF}^{5,12}$.

En relación con los microorganismos aislados, la mayor parte de los trabajos refieren a las cocáceas grampositivas como los principales causantes ${ }^{1,5,13}$, nuestro estudio es concordante con la literatura científica ya que la especie más frecuente fue $S$. aureus, encontrando también Staphylococcus coagulasa negativa (SCN) y Streptococcus spp. En cuanto a las bacterias gramnegativas encontramos a E. coli, K. pneumoniae y Salmonella spp entre otros, reiterando que son especies a ser consideradas en los pacientes con $\mathrm{NF}^{1,5,14}$. El compromiso por virus o bacterias atípicas se encontró en cuatro pacientes en esta cohorte.

El tratamiento antimicrobiano empírico fue establecido por protocolo de la institución recibiendo la mayoría de ellos, piperacilina/tazobactam $(87,6 \%)$; en diferentes estudios se ha demostrado que éste es un antimicrobiano eficaz y seguro en infecciones graves en la edad pediátrica, incluyendo la NF y, al ser comparado con otros antimicrobianos de amplio espectro para esta patología, brinda adecuada protección con poca evidencia de falla terapéutica ${ }^{5,15-17}$. Los demás antimicrobianos se prescribieron según el foco infeccioso que tuviesen los pacientes. No se realizó una categorización por grupos de riesgo para dar un manejo selectivo a cada uno de ellos.

La terapia antimicrobiana final empleada fue diversa, incluyendo cambio a fármacos de segunda línea, ya fuese por evolución clínica desfavorable (en 18 ocasiones) o por aislamientos microbiológicos en los cuales se logró hacer una terapia dirigida (en 8 episodios); el más frecuentemente indicado fue meropenem que se usó en $18 \%$ de los pacientes. En la literatura médica se menciona este antimicrobiano como adecuado para el tratamiento de infecciones graves, siendo ideal como agente de segunda línea en pacientes sin aislamiento microbiológico que no mejoran con la terapia empírica inicial, gracias a su excelente perfil como antibacteriano y por sus propiedades farmacocinéticas y farmacodinamicas ${ }^{18-20}$. Otros antimicrobianos utilizados en segunda intención fueron vancomicina -el más empleado- inicio conjunto de antimicóticos como fluconazol y voriconazol, o adición de antivirales, en este caso aciclovir y ganciclovir/ valganciclovir, Todos estos medicamentos son útiles en el tratamiento de la NF pero sólo se deben indicar en pacientes que cumplan ciertos criterios o en los que se identifiquen microorganismos que lo requieran $n^{4,5,11,12,21}$.

La mortalidad en los episodios descritos fue de 6,7\%, similar a lo reportado en la literatura médica latinoamericana $^{2,3}$.

El recuento inicial de neutrófilos fue, en promedio, de 518,4 céls $/ \mathrm{mm}^{3}$ indicando que gran parte de nuestros pacientes tenían neutropenia grave y por ende, gran riesgo de infección ${ }^{2}$. Al final de la hospitalización, la mayoría de nuestros pacientes recuperó los neutrófilos alcanzando, en promedio, a 2.679,7 céls $/ \mathrm{mm}^{3}$. Al asociar los valores de neutrófilos con la mortalidad, encontramos que los pacientes que fallecieron tuvieron un menor recuento de neutrófilos, tanto al inicio como al finalizar tratamiento; en la literatura científica se menciona los bajos recuentos de neutrófilos como criterio de alto riesgo para fallecer ${ }^{8,9,22}$.

El factor estimulante de colonias de granulocitos es considerado un agente útil para acelerar el aumento del recuento de neutrófilos en pacientes con neutropenia ${ }^{23,24}$, y por esta razón es utilizado en los pacientes con NF; en nuestra experiencia se usó en $61,9 \%$ obteniéndose un mayor recuento de neutrófilos al final de la hospitalización.

La media de la PCR en nuestro estudio fue de 113 $\mathrm{mg} / \mathrm{L}$, siendo mayor entre los pacientes que tuvieron hemocultivos positivos. En la literatura médica hay controversia respecto del valor clínico de la PCR en la evaluación de una NF, algunos estudios la consideran relevante o predictor de riesgo de infección grave ${ }^{25,26}$, mientras que otros la descartan como un parámetro importante a la hora de tomar decisiones ya que no ayudaría a predecir los desenlaces clínicos y de laboratorio de los pacientes $^{27,28}$; estimamos, por esta razón, que se necesitan mayores estudios para alcanzar un consenso al respecto.

La principal limitación de nuestro estudio es que por tratarse de un estudio retrospectivo, no se llevó un protocolo estricto para la búsqueda de la etiología, lo que dificulta conocer el número real de pacientes sin precisar la etiología de la NF. Además se impidió realizar asociaciones, nuevos estudios, de diseño prospectivo, favorecerían comprobar las hipótesis aquí propuestas.

En conclusión, encontramos que las características clínicas, microbiológicas y de laboratorio en nuestros niños con NF son similares a las características descritas en la literatura médica en población similar, y gracias a que ya tenemos un mayor conocimiento de nuestra población, podremos tomar mejores decisiones siempre orientadas al beneficio de nuestros pacientes.

\section{Resumen}

Introducción: La neutropenia es una de las complicaciones más comunes en los niños con cáncer y el principal parámetro para determinar el riesgo de infección. Además, en estos pacientes los signos clínicos de infección pueden ser escasos y en ocasiones la fiebre es la única 
manifestación, por lo que todo paciente neutropénico y febril se debe manejar como si presentara una posible infección grave. Objetivo: Describir el comportamiento clínico y de laboratorio de los pacientes con neutropenia febril (NF) atendidos en nuestra institución para racionalizar el manejo futuro de esta complicación. Pacientes y Métodos: Se revisó los registros clínicos acumulados durante un período de 36 meses, de todos los pacientes de 0 a 15 años internados por cáncer y NF. Resultados: En este estudio se encontró el foco infeccioso en 48,6\% de 105 episodios y se logró aislamiento bacteriano por hemocultivos y/o urocultivo en 38\%. Las bacterias encontradas con mayor frecuencia fueron $S$. aureus sensible a meticilina $(20,8 \%)$ y E. coli no productora de BLEE (20,8\%). El antimicrobiano de primera línea más usado fue piperacilina/tazobactam $(87,6 \%)$ y de segunda línea meropenem (18\%). Se usó factor estimulante de colonias de granulocitos en $61,9 \%$ de los pacientes. La mortalidad asociada a estos episodios fue de 6,7\%. Conclusión: Las características clínicas y hallazgos de laboratorio en nuestra institución no difieren mayormente de lo descrito en población pediátrica en otras series.

\section{Referencias bibliográficas}

1.- Solís Y, Álvarez A, Fuentes D, de la Barra D, Avilés C, Becker A, et al. Agentes causantes de bacteriemia en niños con cáncer y neutropenia febril de alto riesgo en seis hospitales de Santiago, Chile, período 2004-2009. Rev Chilena Infectol 2012; 29 (2): 156-62.

2.- Hughes $\mathrm{W} T$, Armstrong D, Bodey G P, Bow E J, Brown A E, Calandra T, et al. Guidelines for the use of antimicrobial agents in neutropenic patients with cancer. Clin Infect Dis 2002; 34: 730-51.

3.- Paganini H. Enfoque clínico y tratamiento de los niños con neutropenia y fiebre. Arch Argent Pediatr 2007; 105 (3): 225-35.

4.- Almeida A, Sapolnik R, Mendonça N. New guidelines for the clinical management of febrile neutropenia and sepsis in pediatric oncology patients. J Pediatr (Rio J) 2007; 83: 54-61.

5.- Paganini H, Santolaya M. Diagnóstico y tratamiento de la neutropenia febril en niños con cáncer. Rev Chilena Infectol 2011; 28 (Supl 1): S10-S38.

6.- Lindblom A, Bhadri V, Söderhäll S, Ohrmalm L, Wong M, Norbeck O, et al. Respiratory viruses, a common microbiological finding in neutropenic children with fever. J Clin Virol. 2010; 47 (3): 234-7.

7.- Comité Nacional de Infectología Pediátrica. Consenso sobre el cuidado del paciente oncológico neutropénico febril. Actualización 2008-2009. Arch Argent Pediatr 2010; 108, 2 : 47-70.

8.- Arnello L M, Quintana B J A, Barraza C P. Neutropenia febril en niños con cáncer en un centro asistencial en Santiago, Chile. Rev Chilena Infectol 2007; 24, 1: 27-32

9.- Hakim H, Flynn P M, Srivastava D K, Knapp K M, Li C, Okuma J, et al. Risk prediction in pediatric cancer patients with fever and neutropenia. Pediatr Infect Dis J. 2010; 29 (1): 53-9.

10.- Vargas L. Cáncer en pediatría. Rev Chil Pediatr
2000; 71 (4): 40-7.

11.- Ammanna R, Tissingb W, Phillipsc B. Rationalizing the approach to children with fever in neutropenia. Curr Opin Infect Dis 2012, 25: 258-65

12.- López P, López E. Neutropenia febril en pediatría. Infectio 2008; 12 (1): 290-7.

13.- Greenberg D, Moser A, Yagupsky P, Peled N, Hofman Y, Kapelushnik J, et al. Microbiological spectrum and susceptibility patterns of pathogens causing bacteremia in paediatric febrile neutropenic oncology patients: comparison between two consecutive time periods with use of different antibiotic treatment protocols. Int J Antimicrob Agents 2005; 25 : 469-73.

14.- Hakim H, Flynn P M, Knapp K M, Srivastava D K, Gaur A H. Etiology and clinical course of febrile neutropenia in children with cancer. J Pediatr Hematol Oncol 2009; 31 (9): 623-9.

15.- Wolf M, Simon A. The use of piperacillintazobactam in neonatal and paediatric patients. Expert Opin Drug Metab Toxicol 2009; 5, 1: 57-69.

16.- Uygun V, Tezcan G, Ogunc D, Yesilipek A, Hazar V. Piperacillin/tazobactam versus cefepime for the empirical treatment of pediatric cancer patients with neutropenia and fever: a randomized and open-label study. Pediatr Blood Cancer 2009; 53: 610-4.

17.- Vural S, Erdem E, Gulec S, Yidilmark Y, Kebudi R. Imipenem - cilastatin versus piperacillin-tazobactam as monotherapy in febrile neutropenia. Pediatr Int 2010; 52: 262-7.

18.- Kollef M H. Update on the appropriate use of meropenem for the treatment of serious bacterial infections. Clin Infect Dis 2008; 47: 1-2.

19.- Baldwin C M, Lyseng-Williamson K A, Keam S J. Meropenem: a review of its use in the treatment of serious bacterial infections. Drugs 2008; 68 (6): 803-38.

20.- Müller J, Garami M, Constantin T, Schmidt M, Fekete G, Kovács G. Meropenem in the treatment of febrile neutropenic children.

Pediatr Hematol Oncol. 2005; 22 (4): 277-84.

21.- Cortez D, Rodríguez N, Benadof D, Zamorano A, Tordecilla J. Bacteriemia en pacientes oncológicos. Experiencia en un hospital pediátrico. Rev Chilena Infectol 2012; 29 (2): 164-8.

22.- Baorto E P, Aquino V M, Mullen C A, Buchanan G R, DeBaun M R. Clinical parameters associated with low bacteremia risk in 1100 pediatric oncology patients with fever and neutropenia. Cancer 2001; 92: 909-13.

23.- Clark O A, Lyman G, Castro A A, Clark L G, Djulbegovic B. Colony stimulating factors for chemotherapy induced febrile neutropenia. Cochrane Database Syst Rev 2003; 3 : CD003039.

24.- Ozkaynak F, Krailo M, Chen K, Feusner J. Randomized comparison of antibiotics with and without granulocyte colony-stimulating factor in children with chemotherapy-induced febrile neutropenia: a report from the children's oncology group. Pediatr Blood Cancer 2005; 45: 274-80.

25.- Santolaya M E, Álvarez A M, Becker A, Cofré J, Enríquez N, O’Ryan M, et al. Prospective, multicenter evaluation of risk factors associated with invasive bacterial infection in children with cancer, neutropenia and fever. J Clin Oncol; 2001, 19, 14: 3415-21.

26.- Santolaya M, Cofré J, Beresi V. C-reactive protein: valuable aid for the management of febrile children with cancer and neutropenia. Clin Infect Dis 1994; 18: 589-95.

27.- Jeddi R, Achour M, Amor R B, Aissaoui L, Bouterâa W, Kacem K, et al. Factors associated with severe sepsis: prospective study of 94 neutropenic febrile episodes. Hematology; 2010; 15, 1: 28-32.

28.- Kassam A, Chan A, Dzolganovski B, Constantin J, Ramphal R, Grant R, et al. No association between protein $\mathrm{C}$ levels and bacteremia in children with febrile neutropenia. J Pediatr Hematol Oncol 2009; 31: 647-50. 